\title{
Die therapeutische Wirksamkeit westlicher Atemtherapiemethoden: Ein systematischer Review
}

\author{
Roger Stutz ${ }^{a}$ Delia Schreiber ${ }^{b}$ \\ a Basel, Schweiz; \\ ${ }^{\mathrm{b}}$ Männedorf, Schweiz
}

\section{Schlüsselwörter}

Angst - Asthma - Atemtherapie - Bluthochdruck .

Burnout · Depression · Herzinfarkt · Rückenschmerzen

\section{Zusammenfassung}

Dieser systematische Review untersucht und beurteilt die Wirksamkeit von verschiedenen westlichen Atemtherapiemethoden auf somatische und psychische Parameter und eventuelle negative Auswirkungen atemtherapeutischer Interventionen auf Patienten. 23 Studien zu den Methoden nach Middendorf, Richter, Dürckheim/Graubner, Papworth, van Dixhoorn und Buteyko sowie 1 Review zur Buteyko-Methode wurden tabellarisch qualitativ bewertet und miteinander verglichen. Eine Vielfalt von vor allem somatischen, aber auch psychischen Parametern der Studien wurde untersucht. Es zeigten sich klare Hinweise auf die Wirksamkeit vor allem in Bezug auf Asthma, Rückenbeschwerden, Herzdysfunktionen und Schmerzen, aber auch auf verschiedene psychische Parameter, vor allem Angstzustände. Atemtherapie verändert die Lungenfunktion kaum, allerdings, bei signifikant verbesserten Endparametern, Asthma und Herzdysfunktion. Die somatische und die psychische Wirksamkeit der atemtherapeutischen Funktionen hielten sich im vorliegenden Review die Waage. Atemtherapeutische Interventionen sind sicher. Wie bisherige Studien zeigen, sind bei qualifizierter Diagnosestellung und kompetenter Arbeitsweise in der Regel keine Nebenwirkungen zu erwarten. Es gibt Hinweise, dass atemtherapeutische Methoden einen direkten psychotherapeutischen Effekt haben können. Hier sind weitere kontrollierte Interventionsstudien nötig, ebenso bei den Parametern, die die größte Behandlungssensibilität versprechen, wie Asthma, Lebensqualität bei Asthma, Rückenbeschwerden, Herzdysfunktion, Angst und Depression. Sorgfältig designte Interventionsstudien könnten hier auch in Zukunft gute Effekte zeigen.

(c) 2017 S. Karger GmbH, Freiburg

\author{
Keywords \\ Anxiety - Asthma - Breathing therapy - Hypertension . \\ Burnout - Depression - Cardiac dysfunction - Back pain
}

\section{Summary \\ The Effectiveness of Occidental Breathing Therapy \\ Methods: A Systematic Review}

This systematic review investigates and evaluates the efficacy of different Western methods of breathing therapy with regard to somatic and psychological parameters, as well as possible adverse effects of breathing therapy. 23 studies on the methods according to Middendorf, Richter, Dürckheim/Graubner, Papworth, van Dixhoorn and Buteyko, as well as 1 review on the Buteyko method, were arranged in tabular form and then compared and evaluated. A variety of mainly somatic but also psychological parameters were examined in the studies. There were clear indications of the efficacy of breathing therapy, especially regarding asthma, chronic back pain, cardiac dysfunctions, and pain. There were also indications that breathing therapy can be effective in the treatment of various psychological parameters, in particular, anxiety. Breathing therapy hardly changes the lung functions, but it can have a clinically significant effect on asthmatic symptoms and cardiac dysfunctions. In our review, the somatic and the psychological effectiveness of breathing therapy balanced each other. Breathing therapy is safe. As previous studies have shown, no negative side effects are to be expected as long as a qualified diagnostic system is used and breathing therapy is applied in a competent and professional manner. There are indications that therapeutic breathing methods can have a direct psychotherapeutic effect. More controlled interventions studies are needed to obtain additional evidence of such positive effects. The same is true for those parameters that lead to positive treatment results after breathing therapy in cases of asthmatic symptoms or regarding the quality of life of asthma patients, chronic back pain, certain cardiac dysfunctions, anxiety, and depression. In these cases, future, carefully designed intervention studies could also show positive effects.

\section{KARGER \\ Fax +497614520714

() 2017 S. Karger GmbH, Freiburg 


\section{Einleitung}

\section{Definition des Begriffs Atemtherapie}

Atemtherapie umfasst ein breites Spektrum von Methoden, die in vielfältigen Settings angewendet werden [1-10]. Mehling ([10], p 158) beschreibt die Atemtherapie wie folgt:

«Atemtherapie im weitesten Sinne könnte verstanden werden als jegliche Therapieform, die primär über die Arbeit am Atem einen therapeutischen Effekt bewirken möchte.»

Einerseits gibt es die «klassischen» Atemtherapiemethoden wie z.B. nach Middendorf [1-3, 7, 9-13], Glaser [9], Richter [14], van Dixhoorn [15] und Wolf $[9,16]$, bei denen der Atem als ein auf die Ganzheit des Menschen (körperlich, seelisch, geistig) einwirkendes Element betrachtet wird. Daneben gibt es die eher funktionalen mechanischen Atemtherapieverfahren wie z.B. die Atemphysiotherapie, das Atemmuskeltraining, das «inspiratory muscle training», die stimulierende Spirometrie (incentive spirometry), die Atemtherapie nach Schroth [17] oder die ButeykoAtemtechnik [18].

Auch in den Disziplinen der Logopädie und der Stimmbildung [19] nimmt die Atmung einen wichtigen Stellenwert ein. Zu nennen sind auch das Rebirthing nach Orr und das holotrope Atmen nach Grof, die funktionelle Entspannung nach Fuchs oder die körperpsychotherapeutischen Methoden wie z.B. die Atemtherapie nach Reich, die Bioenergetik nach Lowen oder die Atem- und Körperpsychotherapie nach Bischof [20, 21].

Ein weiterer wichtiger Bereich umfasst die apparativen Atemtherapieverfahren, wie z.B. die Inhalationstherapien oder die künstliche Beatmung.

Ein weiterer Bereich umfasst die zahlreichen Atempraktiken und Atemübungen in den verschiedenen Yogaarten (Pranayama), beim Tai-Chi und Qigong oder die Atemmeditation als Teil der buddhistischen Achtsamkeitsmeditation (Vipassana).

\section{Ziele}

Ziel dieser Übersichtsarbeit war die systematische Erfassung der wissenschaftlichen Literatur zur Atemtherapie unter Berücksichtigung folgender Schwerpunkte:

1) Beurteilung des Nutzens von methodenspezifischen atemtherapeutischen Interventionen.

2) Feststellen von möglichen negativen Auswirkungen.

\section{Methodik}

Bei der methodischen Vorgehensweise haben wir uns an der von der evidenzbasierten Medizin ausgearbeiteten Leitlinie «PRISMA - Transparent Reporting of Systematic Reviews and Meta-Analyses» [22], der von Both [23] erarbeiteten Richtlinie «Brimful of STARLITE: towards standards for reporting literature searches» und des «Cochrane Handbook for Systematic Reviews of Interventions» [24] orientiert.
Auswahl der Studien, Datenauszug und Management

Beide Autoren überprüften unabhängig voneinander die Suchergebnisse nach infrage kommenden Studien und extrahierten unabhängig voneinander die Daten in einer standardisierten Form (Abb. 1). Abweichungen wurden durch Konsens gelöst.

Beurteilung der Gefahr der Verzerrung (Risk of Bias) bei den eingeschlossenen Studien

Es war geplant, die Verzerrungsgefahr mit dem Instrument «risk of bias» von Cochrane [24] auszuwerten. Da es jedoch schwer möglich ist, in Studien dieser Art Therapeuten oder Ärzte zu anonymisieren, wurden diese Kriterien nicht berücksichtigt. Daher sollten beide Autoren unabhängig voneinander für jede Studie eine Beurteilung des Verzerrungsrisikos erstellen. Sollte das Verzerrungsrisiko einer Studie unklar sein, würde der Autor der Studie befragt werden. Als Biasrisiken galten im Rahmen dieses Reviews z.B. Verzerrungen aufgrund von Dropout-Fehlerberechnungen, Bestätigungsfehlern, fälschlicher Kausalzusammenhänge und Behandlerbias (wenn der Behandler gleichzeitig die Studie durchführt).

\section{Suchstrategie zur Auswahl der Studien}

Datenbanken

Die nachfolgenden Datenbanken wurden systematisch nach wissenschaftlichen Publikationen über Atemtherapie durchsucht:

- PubMed/Medline

- CAMBASE (Complementary and Alternative Medicine)

- PsycINFO

- wissenschaftliche Datenbank der European Association for Body Psychotherapy (EABP)

- Deutsche Nationalbibliothek

- Google Scholar

Suchbegriffe

Für die Suche nach wissenschaftlichen Publikationen wurden folgende Schlagworte verwendet:

- Atemtherapie

- Atemgymnastik

- Atemübungen

- Atempsychotherapie

- Körperpsychotherapie

- Breathing Therapy

- Breathing Exercises

- Body Psychotherapy

Die Schlagworte wurden teilweise mit den Boole'schen Operatoren 'AND' und/oder 'OR' verknüpft, außerdem wurden Platzhalter $\left.{ }^{*}\right)$ als Stellvertreter für einen Buchstaben oder eine Buchstabengruppe verwendet.

Bei der Suche in PubMed/Medline wurde mit den von der United National Library of Medicine verwalteten MeSH Terms (Medical Subject Headings) und Subheadings gearbeitet. Da bei der Publikationssuche mit den MeSH Terms zahlreiche relevante Studien nicht unter den Treffern aufgelistet wurden, wurde zusätzlich auch mit der Schlagwortsuche gearbeitet. Dabei wurde der 
Abb. 1. Flussdiagramm eingeschlossene und ausgeschlossene Studien (Flow Diagram PRISMA Statement 2009).

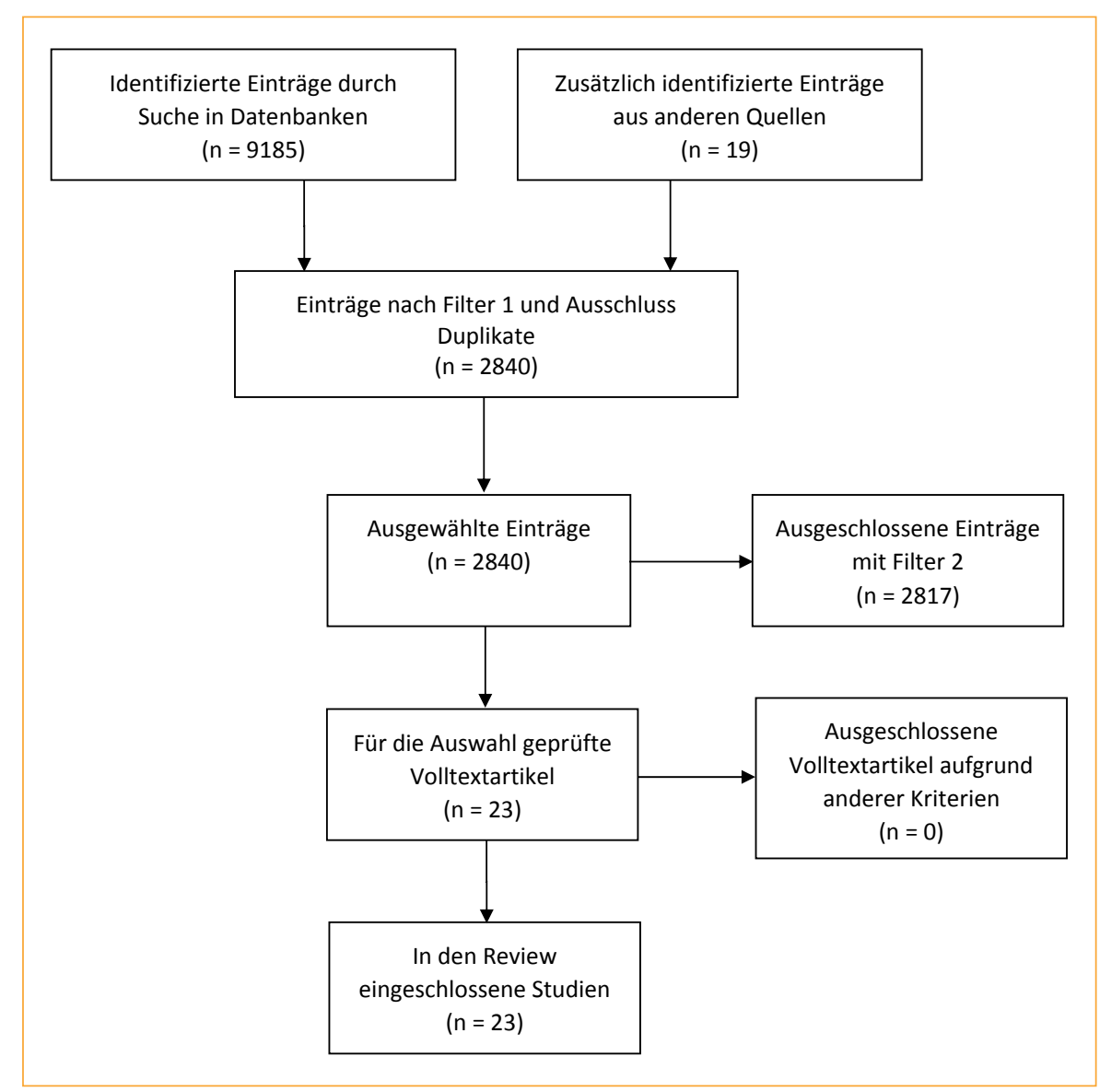

Suchbegriff «breathing therapy» mit «breathing exercises» kombiniert, da beim Begriff «breathing therapy» viele Studien (104 064) in der Ergebnisliste erschienen, die sich auf eine rein apparative atemtherapeutische Anwendung (Inhalationstherapien, respiratorisches Biofeedback, «intermittent positive pressure breathing» usw.) bezogen. Zusätzlich wurden die in der Datenbank PubMed vorhandenen Filter (Clinical Trial, Review, Publikationszeitraum, Humans) verwendet, um die Suchergebnisse der Publikationen auf die gestellte Fragestellung einzugrenzen.

Bei der Suche in der Datenbank Google Scholar wurden die Suchbegriffe «Atemtherapie» bzw. «Atemgymnastik» mit «Atemübungen» und mit «Studie» kombiniert, da viele Treffer (3340) in den Ergebnissen aufgelistet wurden, die keine klinischen Studien oder Reviews waren oder die sich auf eine apparative Anwendung der Atemtherapie bezogen. Dasselbe Vorgehen wurde bei den englischen Suchbegriffen «breathing therapy» und «breathing exercises» und «study» angewendet (1950 Treffer).

Der Suchbegriff «Körperpsychotherapie» wurde mit «Atem» bzw. «Atemtherapie» und «Studie» verknüpft, um auch hier die Trefferzahl auf die gestellte Fragestellung zu beschränken.

Die detaillierte Liste mit den verwendeten Suchbegriffen bzw. Suchbegriffsgruppen, den verwendeten Filtern, den Trefferzahlen und dem jeweiligen Suchdatum wurde in Suchprotokolllisten aufgeführt. Diese Daten können in der Langversion dieses Reviews unter www.atem.ch und unter www.deliaschreiber.ch nachgelesen werden.

\section{Einschlusskriterien}

Arten von Studien: Es wurden alle Arten von klinischen Studien sowie Reviews und Meta-Analysen, die in englischer oder deutscher Sprache erhältlich waren und zwischen dem 1. Januar 1980 und dem 31. Dezember 2014 publiziert wurden, eingeschlossen.

Arten von Teilnehmern: Kinder, Jugendliche und erwachsene Personen mit klinischer Diagnose konnten ausgewählt und in den Review aufgenommen werden.

Arten von Interventionen: Es wurden Studien in den Review aufgenommen, bei denen Atemtherapiemethoden bzw. atemtherapeutische Interventionen angewendet wurden, die einem klar definierten methodisch-therapeutischen Behandlungskonzept zugeordnet werden konnten. Dabei wurden alle Arten von Ateminterventionen wie z.B. Atemübungen, Atembehandlungen und Atemmeditation in den Review einbezogen.

\section{Ausschlusskriterien}

Nichtklinische oder nichtexperimentelle Studien, Kongressberichte, Fallberichte sowie Studien, bei denen ausschließlich methodenunspezifische atemtherapeutische Interventionen angewendet wurden (slow breathing, deep breathing, Zwerchfellatmung, pursed lips breathing usw.) - ohne methodisch-therapeutisches Gesamtkonzept - wurden ausgeschlossen.

Ebenso ausgeschlossen wurden klinische Studien und Reviews, in denen apparative atemtherapeutische Anwendungen (respiratorisches Biofeedback, «device-guided breathing», Inhalationsthera- 
Tab. 1. Studiendesigns der in den Review aufgenommenen Studien

\begin{tabular}{|c|c|c|c|c|c|}
\hline Methode & $\begin{array}{l}\text { Interventionsstudien } \\
\text { ohne Kontrollgruppe }\end{array}$ & $\begin{array}{l}\text { Kontrollierte } \\
\text { Interventionsstudien }\end{array}$ & $\begin{array}{l}\text { Randomisierte, } \\
\text { kontrollierte } \\
\text { Interventionsstudien }\end{array}$ & Reviews & Total \\
\hline Middendorf & 2 & 4 & 2 & 0 & 8 \\
\hline Richter/Dürckheim & 1 & 0 & 0 & 0 & 1 \\
\hline van Dixhoorn & 0 & 0 & 5 & 0 & 5 \\
\hline Papworth & 0 & 0 & 1 & 0 & 1 \\
\hline Buteyko & 0 & 1 & 6 & 1 & 8 \\
\hline Total & 3 & 5 & 14 & 1 & 23 \\
\hline
\end{tabular}

pien, Überdruckbeatmung usw.) untersucht wurden. Auch ausgeschlossen wurden physiotherapeutische Ateminterventionen wie z.B. «inspiratory muscle training» und stimulierende Spirometrie. Weiter ausgeschlossen wurden alle Formen von Yogapraktiken (Asanas) und Yoga-Atemübungen (Pranayama) sowie Studien über Qigong und Tai-Chi. Studien, bei denen die Intervention aus Meditieren bestand, wurden in den Review aufgenommen, wenn der Atem während der Meditation im Fokus der Aufmerksamkeit stand. Hingegen wurden alle Studien ausgeschlossen, bei denen das Meditieren keinen Bezug zum Atem hatte. Weiter wurden alle Studien ausgeschlossen, bei denen die Interventionen aus mehreren Anwendungsmethoden bestanden, wenn diese innerhalb derselben Interventionsgruppe gleichzeitig oder nacheinander angewendet wurden und keine Interventionsgruppe existierte, deren Probanden ausschließlich atemtherapeutische Interventionen erhielten, da bei solchen Studiendesigns nicht eindeutig eruierbar ist, welche Wirkung die atemtherapeutische Intervention erzielte. Interventionen ohne Atemübungen oder Atemübungen, die keine Schlüsselstellung in der Therapie einnahmen, wurden ebenso ausgeschlossen.

\section{Ergebnisse}

\section{Suchergebnisse}

Von den insgesamt 9204 Studien wurden 9181 ausgeschlossen (Abb. 1).

\section{Qualitative und quantitative Ergebnisse}

Studiendesigns der in den Review aufgenommenen Studien

Von den 23 in den Review aufgenommenen Studien [18, 19, 25-45] sind 14 randomisierte, kontrollierte Interventionsstudien [26, 28, 29, 32, 33, 36-39, 41-45] (davon sind 2 randomisierte, kontrollierte Doppelblindstudien [26, 39], 1 eine randomisierte, kontrollierte einfach-verblindete Studie [37]), 5 kontrollierte Interventionsstudien $[18,19,25,30,35], 3$ Interventionsstudien ohne Kontrollgruppe [31, 34, 40] und 1 ein Review [27].

Tabelle 1 zeigt die Studiendesigns aufgeschlüsselt in die einzelnen Atemtherapiemethoden.

\section{Methodische Qualität der Studien}

$\mathrm{Da}$ in den Studien dieses Reviews sehr verschiedene somatische, psychische und andere Endparameter gemessen wurden und auch
Tab. 2. Methodische Qualität der Studien

\begin{tabular}{lcc}
\hline Qualitätsgrad der Studie & Anzahl & Anteil \\
\hline $0=0-4$ Punkte: indiskutabel & 0 Studien & $0 \%$ \\
$1=5-8$ Punkte: ungenügend & 1 Studie & $4,7 \%$ \\
$2=9-12$ Punkte: genügend & 7 Studien & $33,3 \%$ \\
$3=13-16$ Punkte: gut & 13 Studien & $62 \%$ \\
$4=17-20$ Punkte: sehr gut & 0 Studien & $0 \%$ \\
\hline Total & 21 Studien & $100 \%$ \\
\hline
\end{tabular}

die atemtherapeutischen Interventionen nicht einheitlich waren, wurde der Vergleich durch die hohe Diversität erschwert. Angestrebt wurde eine gewisse Vereinheitlichung, um die in den Studien nachgewiesene Wirksamkeit vergleichbar machen zu können. Auch wurde angestrebt, die Qualität der Studien ersichtlich zu machen. Hierzu erstellten die Autoren einen Fragenkatalog mit 7 Qualitätskriterien und beurteilten die einzelnen Studien anhand des nachfolgenden Rasters. Abweichende Einschätzungen wurden durch Konsens gelöst.

- Ist die Studie kontrolliert, randomisiert/stratifiziert, verblindet, und finden die Interventionen der einzelnen Gruppen gleichzeitig statt? (je 1 Punkt);

- Wie hoch ist die Biasgefahr? (klein = 3 Punkte; mittel = 2 Punkte: groß = 1 Punkt);

- Wie sinnvoll sind die Intervention und ihre Dauer? (sinnvoll = 3 Punkte; sinnvoll, aber mit Abstrichen = 2 Punkte; Dauer oder Intervention sind wenig sinnvoll = 1 Punkt; weder Intervention noch Dauer machen Sinn = 0 Punkte);

- Wie sind die Qualität und Quantität der Probanden? (genügend Probanden für $80 \%$ Power $=1$ Punkt; mehr Probanden $=2$ Punkte; Art der Rekrutierung senkt die Biasgefahr = 1 Punkt; kontrollierte Homogenität mit der Kontrollgruppe $=1$ Punkt);

- Wie wurde mit Dropouts verfahren? (Dropouts werden nicht erwähnt $=0$ Punkte; Dropouts werden lediglich erwähnt $=1$ Punkt; Dropoutdaten werden mit intent to treat berechnet $=2$ Punkte);

- Messen die verwendeten Messinstrumente, was sie messen sollen? Werden sie sinnvoll angewendet? (sinnvolle Messinstrumente = 1 Punkt; sinnvolle Anwendung = 1 Punkt);

- Misst die Studie die Nachhaltigkeit der Intervention? (wird nicht gemessen $=0$ Punkte; wird nach 3 Monaten gemessen = 1 Punkt; wird nach 6 Monaten und später gemessen $=1$ Punkt). 


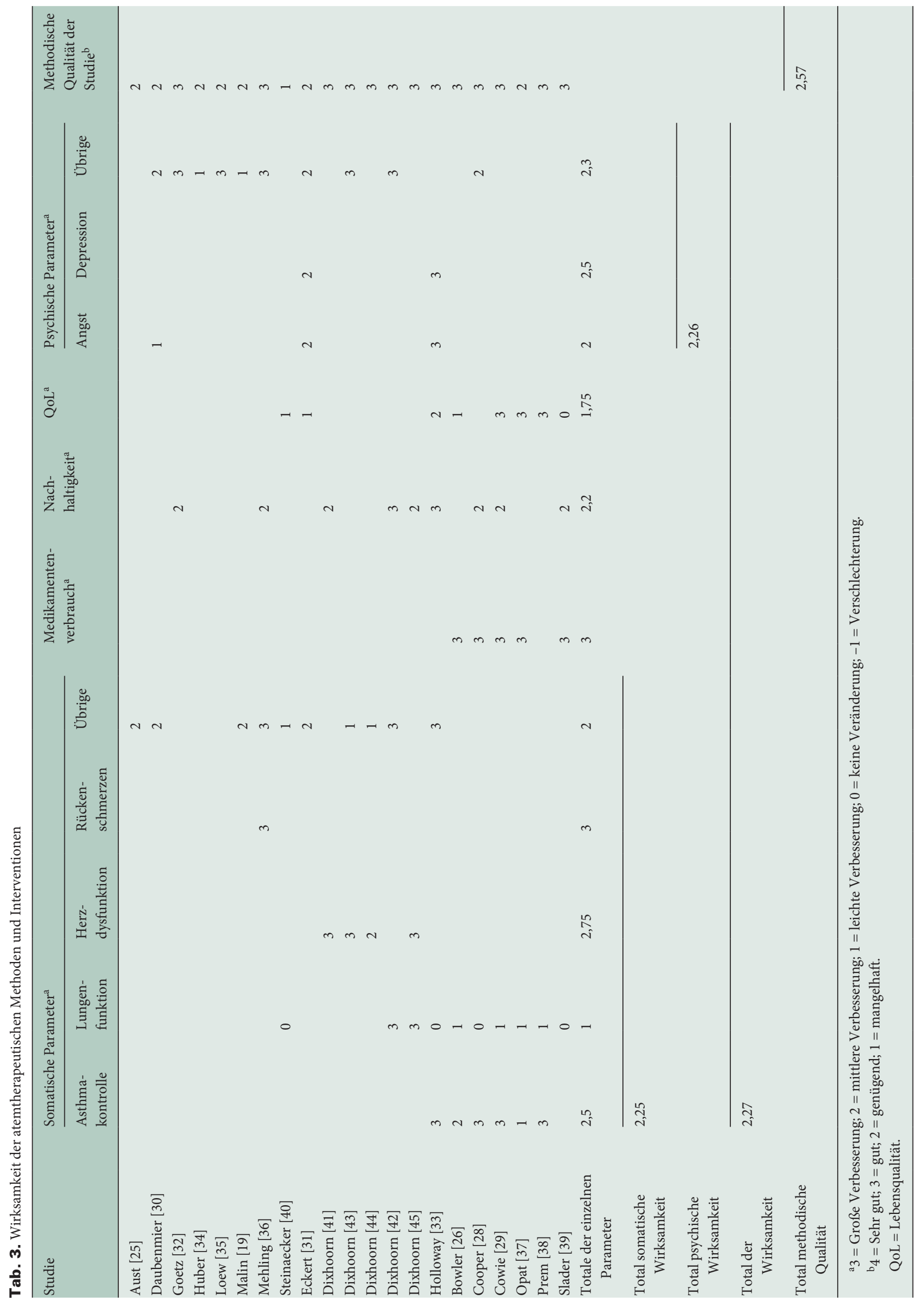


Nach Addition dieser Qualitätspunkte konnten die Studien theoretisch eine Punktzahl zwischen 0 und 20 erreichen. Die qualitative Bewertung der Studien ist in Tabelle 2 und Tabelle 3 dargestellt.

Wirksamkeit der atemtherapeutischen Methoden und Interventionen

Um die Wirksamkeit der atemtherapeutischen Interventionen numerisch zu beurteilen, gingen die Autoren nach folgenden Kriterien vor: Punkteverteilung (siehe Tab. 3) aufgrund der Aufteilung in somatische und psychische Parameter und deren Veränderungen, ferner Veränderung im Medikamentenverbrauch, subjektiv empfundene Lebensqualität und Nachhaltigkeit einer möglichen positiven Wirkung über mehrere Wochen oder Monate (3-stufiges Modell: vor und nach 3 Monaten und nach 6 oder mehr Monaten). Die Autoren beurteilten jede Studie einzeln. Abweichende Einschätzungen wurden durch Konsens gelöst, allenfalls wurden weitere Experten hinzugezogen.

Tabelle 3 zur Wirksamkeit der atemtherapeutischen Interventionen zeigt in numerischer Form die Einschätzung der Veränderung bzw. der Verbesserung der in den Studien gemessenen somatischen, psychischen und übrigen Parameter. Die «übrigen somatischen Parameter» beziehen sich auf Singstimme, Körpergleichgewichtsreaktion, Genauigkeit der Atemwahrnehmung, Körperwahrnehmung, nicht genauer definierte allgemeine somatische Symptome, Ventrikelfibrillation, Durchblutungsstörungen, Toleranz gegenüber körperlichem Training bei Herzpatienten und Entspannungsfähigkeit. Unter «übrige psychische Parameter» haben wir die Affektregulation und die Sammlungsfähigkeit auf den Atem zusammengefasst, und hinsichtlich der Burnoutgefährdung: emotionale Abgrenzungsfähigkeit, mentale Stabilität, subjektives Erleben von sozialer Unterstützung, offensive Bewältigungsstrategie bei Problemen, innere Ruhe und Ausgeglichenheit, Perfektionsstreben, Verausgabungsbereitschaft und Resignation bei Misserfolgen.

Die methodische Qualität ergibt sich aus den Einschätzungen von Tabelle 2.

\section{Ergebnisse}

23 Studien zu den Methoden nach Middendorf [19, 25, 30, 32 , 34-36, 40], Richter [31], Dürckheim/Graubner [31], Papworth [33], van Dixhoorn [41-45] und Buteyko [18, 26, 28, 29, 37-39] sowie 1 Review [27] zur Buteyko-Methode wurden tabellarisch qualitativ bewertet und einander in ihren Endparametern, sofern diese deckungsgleich waren, gegenübergestellt und miteinander verglichen. Um die Vergleichbarkeit der Veränderungen der Parameter durch die Interventionen zu erzielen, wurden die Resultate anhand definierter Kriterien neu eingeschätzt.

Eine Verschlechterung (-1) der Symptome im Rahmen der atemtherapeutischen Interventionen kam in keiner der Studien vor. Wohl beschrieben einige Studien vereinzelte Exazerbationen $[26,28,36-38,43]$, jedoch verteilten sich diese gleichmäßig auf Interventions- und Kontrollgruppen.

Das Total der Wirksamkeit der atemtherapeutischen Behandlungen zeigt im Durchschnitt aller Parameter mit dem Wert 2,27 eine mittlere bis gute Verbesserung. Diese teilte sich gleichmäßig auf zwischen somatischer $(2,25)$ und psychischer Wirksamkeit $(2,26)$.

Der höchste Verbesserungswert (3) konnte bezüglich des Medikamentenverbrauchs bei Asthmapatienten [26, 28, 29, 37, 39] erreicht werden sowie bei Rückenschmerzen [36].

Ebenfalls gute bis sehr gute Werte $(2,5)$ erreichte die Asthmakontrolle im Rahmen der Buteyko-Interventionen [26, 28, 29, 33, 38]. Die Lungenfunktionsparameter wurden dabei von den atemtherapeutischen Interventionen kaum beeinflusst.

Die van Dixhoorn-Methode [41-45] (vom methodischen Ansatz her verwandt mit der Middendorf-Methode [19, 25, 30, 32, $34-36,40])$ zeigte eine mittlere bis große Verbesserung der Herzdysfunktion $(2,75)$.

Die Nachhaltigkeit der Interventionen insgesamt erreichte den Wert 2,2.

Das Total der methodischen Qualität $(2,57)$ ist genügend bis gut.

Die Beschreibungen der in diesem Review besprochenen Studien und deren detaillierte Ergebnisse können in der Langversion dieses Reviews unter www.atem.ch und unter www.deliaschreiber. ch nachgelesen werden.

\section{Diskussion}

\section{Somatische Wirksamkeit}

Insgesamt zeigte sich hinsichtlich verschiedener somatischer Parameter eine gute Wirksamkeit der Atemtherapie.

\section{Atemtherapie bei Rückenbeschwerden}

Das sehr gute Resultat zur Verbesserung von Rückenschmerzen [36] wird gestützt durch frühere Studien von Meier [12] und Mehling [7]. In der Studie von Meier [12] gaben 40,6\% der befragten Patienten als Grund für die Inanspruchnahme der atemtherapeutischen Behandlung Rücken- und Gelenkbeschwerden an. 93,7\% der Patienten gaben an, durch die atemtherapeutische Behandlung eine Besserung ihrer Rücken- und Gelenkbeschwerden erfahren zu haben. 44,2\% der befragten Patienten gaben zudem an, dass sie die Anzahl ihrer Arztbesuche nach einer Behandlungsserie von 12 Atemtherapiebehandlungen reduzieren konnten.

Die von Mehling 1999 in Deutschland durchgeführte Studie [7] zeigt, dass $41,1 \%$ der Patienten, die eine Atemtherapie aufsuchten, unter Rücken-/Kreuz-/Ischiasschmerzen, Nacken-/Schultergürtelschmerzen oder unter schmerzhaften Verspannungen litten.

Gemäß den beiden Studien von Meier [12] und Mehling [7] sind Rückenbeschwerden noch vor Atembeschwerden einer der Hauptanlässe, weshalb Patienten eine atemtherapeutische Behandlung in Anspruch nehmen.

\section{Atemtherapie bei Herzdysfunktionen}

Die hier besprochenen Studien zur Wirkung von Atemtherapie bei Herzdysfunktionen [41-45] wurden alle von van Dixhoorn et al. durchgeführt. Er arbeitete zwar mit externen, unabhängigen Ex- 
perten zusammen, untersuchte aber seine eigene Methode, was eine gewisse Biasgefahr birgt. Die Studien sind jedoch sehr sorgfältig designt und durchgeführt und erhielten für ihre methodische Qualität gemäß unseren Kriterien die Note 3 (gut). Sämtliche Studien von van Dixhoorn et al. [41-45] zeigten eine mittlere bis große, klinisch relevante Verbesserung der Herzdysfunktion nach den atemtherapeutischen Interventionen mit mittlerer bis hoher Nachhaltigkeit.

In Bezug auf die Indikation und Wirkung der Atemtherapie bei Herzdysfunktionen zeigt sich auch hier eine Übereinstimmung mit den Resultaten aus der Studie von Meier [12]. In ihrer 2002 durchgeführten Erhebung gaben 21,5\% der befragten Patienten als Grund für die Inanspruchnahme einer Atemtherapie Herzdysfunktionen und Kreislaufprobleme an. Davon gaben 92,9\% der befragten Patienten an, durch die atemtherapeutische Behandlung eine Besserung ihrer Symptome erfahren zu haben.

Auch die Methode von van Dixhoorn [41-45] basiert auf dem Atembewusstsein, also auf dem bewussten Hinlenken der Aufmerksamkeit auf den Atem wie bei der Methode nach Middendorf $[19,25,30,32,34-36,40]$.

Auffallend ist, dass die Teilnehmer der Interventionsgruppen ihren Atem und Körper nachhaltig besser wahrnehmen konnten und sich dabei subjektiv, sowohl physisch als auch psychisch, besser fühlten. Dies war gekoppelt mit einer langsameren Atmung, einem physiologischeren Atemmuster, einem positiven Effekt auf die respiratorische Sinusarrhythmie und die Brachykardie sowie einer signifikanten Verbesserung der ST-Abnormität. Der rein körperlich gesteigerte Trainingseffekt der Kontrollgruppen war nicht gekoppelt mit einer besseren psychischen Befindlichkeit.

van Dixhoorn et al. ließen in nur 2 ihrer Studien [42, 43] psychische Parameter messen, die aber bei den Interventionsgruppen beide Male signifikant waren im Vergleich der Prä-Post-Messung und im Vergleich zur Kontrollgruppe. Zudem beeindruckt der Nachweis der Nachhaltigkeit bis zu 30 Monaten (maximal gemessener Post-Zeitpunkt).

Es scheint, dass die Atemtherapie mit dem Fokus der Aufmerksamkeit auf den Atem und die Entspannung bei den Probanden zu einer gewissen Gewohnheit führt, die den Alltagstransfer übersteht. Auch das reine Leistungstraining zeigte gute Effekte, jedoch war dieses bei den Atemgruppen stärker und mit mehr Wohlbefinden gekoppelt. In Bezug auf die Rehabilitation von Herzpatienten heißt dies, dass eine zusätzliche Atemtherapie zum körperlichen Training gewinnbringend sein kann.

Was die Studien von van Dixhoorn et al. [41-45] vermissen lassen, sind Erhebungen über die Auswirkungen der Atemtherapie auf den Medikamentenverbrauch der Probanden.

\section{Asthmakontrolle und Asthmamedikation}

Keine Veränderung der Messparameter fand sich vor allem in Bezug auf die Lungenfunktion bei Asthmapatienten [28, 39] bzw. nur eine leichte Verbesserung der Lungenfunktion bei bedeutend gesteigerter Asthmakontrolle [26, 33, 37, 38].

Offenbar braucht es für die positive Veränderung der Asthmakontrolle und für eine signifikante Verringerung des Medikamen- tenverbrauchs nicht zwingend eine klinisch bedeutsame Lungenfunktionsverbesserung, sondern vor allem ein verändertes Atemmuster. Es ist bekannt, dass eines der Hauptprobleme bei Asthmapatienten darin besteht, dass sie «zu viel» einatmen und «zu wenig» ausatmen, was zum Hyperventilieren und zu Angstgefühlen führt.

Der Medikamentenverbrauch erreichte eine große Verbesserung $[26,28,29,37,39]$. Er konnte signifikant und klinisch relevant, und wo die Nachhaltigkeit berücksichtigt wurde [28, 29], langfristig gesenkt werden.

Die Methode nach Buteyko [18, 26-29, 37-39] erreichte keine signifikanten Veränderungen in der Lungenfunktion.

In den Studien von van Dixhoorn et al. [41-45] konnten die Probanden vor allem ihre Ruheatemfrequenz signifikant und nachhaltig senken. Die Methode nach van Dixhoorn [41-45] basiert auf dem «Atembewusstsein», also dem bewussten Hinlenken der Aufmerksamkeit auf den eigenen Atem im Sinne der Achtsamkeit. Dies ist auch zentral in allen anderen untersuchten Methoden.

Eine etwas mechanischere Haltung nimmt die Methode nach Buteyko [18, 26-29, 37-39] ein, die nicht den Fokus auf den Atem verlangt, sondern nur Übungen mit dem Atem vorsieht. Mit nur wenig Übung können diese ohne Atemachtsamkeit ausgeführt werden. Die Studien zur Buteyko-Methode [18, 26-29, 37-39] antizipierten denn auch nur eine Veränderung der asthmaabhängigen Lebensqualität und keine davon unabhängige, von den Atemübungen initiierte Stimmungsverbesserung.

Bei Cowie et al. [29] zeigten beide Gruppen nach 6 Monaten eine signifikante Verbesserung ihres Asthmastatus, wobei die Buteyko-Gruppe den Medikamentenverbrauch stärker vermindern konnte. Bei Slader et al. [39] zeigte die Kontrollgruppe einen leicht signifikanten Vorsprung gegenüber der Buteyko-Gruppe in Bezug auf die Asthmasymptome. Beide konnten den Reliever signifikant weniger oft einsetzen.

Cowie et al. [29] bezeichneten die Erfolge der Kontrollgruppe als erstaunlich, zumal die Probanden sich vor allem aus Interesse an der Buteyko-Methode für die Studie gemeldet hätten. Die Autoren betonten auch, dass ein Teil der guten Ergebnisse daher rühren konnte, dass beide Gruppen während $10 \mathrm{~h}$ insgesamt die Gelegenheit hatten, sich zu verschiedenen Aspekten ihrer Asthmaerkrankung zu informieren. Aus der Sicht der Nicht-Buteyko-Atemtherapien erstaunt die Verbesserung in den Kontrollgruppen nicht, da diese hochwirksame Entspannungsatemübungen erhielten, die erfahrungsgemäß mit steigender Übung immer mehr den Ausatem betonen und zu einer Aktivierung des Parasympatikus führen und damit auch angstlindernd wirken.

Insgesamt lässt sich feststellen, dass die Buteyko-Atmung wesentlich zur Reduktion des nachhaltigen Medikamentenverbrauchs bei Asthmapatienten führen kann, dass jedoch die eher atemfokussierten Entspannungsmethoden (Papworth) [33] hinsichtlich der Asthmakontrolle ebenso viel leisten können.

\section{Übrige somatische Parameter}

Die übrigen somatischen Parameter beziehen sich auf die Singstimme [19], Körpergleichgewichtsreaktion [25], Genauigkeit der Atemwahrnehmung [30], Körperwahrnehmung [33, 36, 40, 42], 
nicht genauer definierte allgemeine somatische Symptome [31], Ventrikelfibrillation [41, 44], Durchblutungsstörungen [41], Toleranz gegenüber körperlichem Training bei Herzpatienten [45] und Entspannungsfähigkeit [19, 40, 42]. Die Verbesserungen im Mittel lagen mit einem Wert von 2,0 bei einer mittleren Wirkung.

\section{Lebensqualität}

Die Lebensqualität zeigte vor allem bei Asthmapatienten eine hohe Verbesserung [29, 33, 37, 38].

\section{Wirkung von atemtherapeutischen Interventionen auf die Psyche}

Die psychischen Parameter, die im Rahmen dieses Reviews gemessen und mit atemtherapeutischen Interventionen verbessert werden konnten, sind Angst [30, 31, 33] und Depression [31, 33]. Die Resultate zeigen eine Wirksamkeit von je 1-mal mittel [31] und 1-mal hoch [33].

Aufgeführt in der Kategorie als übrige psychische Parameter (Tab. 3) sind: Affektregulation [19, 30], Sammlungsfähigkeit auf den Atem [28, 30, 36, 42] und hinsichtlich der Burnoutgefährdung $[32,35]$ : emotionale Abgrenzungsfähigkeit, mentale Stabilität, subjektives Erleben von sozialer Unterstützung, offensive Bewältigungsstrategie bei Problemen, innere Ruhe und Ausgeglichenheit, Perfektionsstreben, Verausgabungsbereitschaft und Resignation bei Misserfolgen mit einer mittleren bis hohen Wirksamkeit.

Bemerkenswert ist, dass sich das Total der Wirksamkeit von Atemtherapie in Bezug auf somatische und psychische Wirksamkeit die Waage hält. Die psychischen Parameter wurden vor allem im Rahmen der Middendorf-Methode [19, 25, 30, 32, 34-36, 40] gemessen, da diese Methode in ihrem Konzept davon ausgeht, dass neben der somatischen auch die psychische Befindlichkeit auf die Therapie reagiert.

Mehling et al. [36] zeigten in ihrer Studie, dass sich bei den Probanden der Atemtherapiegruppe neben den Rückenschmerzen auch der Umgang mit Stress und Emotionen signifikant verbesserte. Daubenmier et al. [30] legten eine besonders hinsichtlich der Atem- und Körperpsychotherapie [20, 21] interessante Untersuchung vor. Der von ihnen verwendete 7-Item Body Responsiveness Questionnaire misst bei den Probanden ein zentrales Anliegen der Atem- und Körperpsychotherapie, nämlich inwieweit das Individuum fähig ist, emotionale und körperliche Impulse bewusst wahrzunehmen, sie weder zu unterdrücken noch unkontrolliert auszuagieren, sondern konstruktiv in das eigene Entscheiden und Verhalten zu integrieren im Sinne einer optimierten Emotionsregulation. Verglichen wurden meditationserfahrene und meditationsunerfahrene Probanden. Atemmeditation bezieht sich auf die Tradition, während der Meditationszeit den Atem in den Fokus der Aufmerksamkeit zu stellen und äußere und innere Inhalte, die sich zeigen, zu benennen, jedoch nicht zu bewerten.

Eine Studie von Davidson et al. [46] hat die Hirnaktivität vor und direkt nach einer Achtsamkeitsmeditation und 4 Monate nach einem 8-wöchigen Achtsamkeitsmeditationstraining gemessen. Am Ende der 8-wöchigen Trainingszeit erhielten beide Studien- gruppen eine Grippeimpfung. Die Interventionsgruppe zeigte gegenüber der Kontrollgruppe signifikant höhere Hirnaktivität im linken vorderen Stirnlappen, einer Hirnregion, die für positive Emotionen zuständig ist. Bei diesen Probanden wurden auch signifikant höhere Grippe-Antikörpertiter nachgewiesen. Je höher die Aktivität im linken vorderen Stirnlappen war, umso höher war der Antikörpertiter. Diese Resultate könnten ein Mediatormodell für die Atemtherapie mit Atemachtsamkeitsfokus andeuten. Durch die Atemarbeit wird der Nervus vagus aktiviert. Gleichzeitig verändert sich die Hirnaktivität positiv, was biochemische Prozesse auslöst, die die vielfach gemessene, verstärkt positive psychische Befindlichkeit erklären könnte. Diese wiederum kann zu salutogenen somatischen Prozessen führen.

In einer Untersuchung von van Dixhoorn et al. [43] wird nachgewiesen, dass der Zugewinn an positiver Gemütslage nicht abhängig ist vom physischen Trainingseffekt, der die Herzdysfunktion verbessern konnte.

Atemtherapie ist grundsätzlich eine Therapieform, die die Selbstwirksamkeitserwartung von Patienten fördert, indem sie demonstriert, wozu der Organismus unter bewusstem Einbezug des Atems fähig ist, und zum selbstständigen Üben auffordert. Diese empirische Beobachtung müsste jedoch mit Studien zur Selbstwirksamkeitserwartung im Rahmen der Atemtherapie nachgewiesen werden.

Es muss angemerkt werden, dass zum Zeitpunkt der Studienauswahl international nur 23 Studien vorhanden waren, die einem klar definierten methodisch-therapeutischen Behandlungskonzept zugeordnet werden konnten. Diese Anzahl ist nicht ausreichend für eine Meta-Analyse, zudem zeigte sich eine hohe Heterogenität der untersuchten Zielparameter und der angewandten Methoden und Methodik. Die Auswertung im vorliegenden Review erfolgte demnach, wenn auch systematisch, auf rein qualitativer Ebene. Zudem waren bei einigen Studien [19, 31, 40-45] die bei der Studiendurchführung mitbeteiligten Personen gleichzeitig die Behandler. Diese Tatsache wurde jedoch in der Qualitätseinschätzung der Studien als Bias berücksichtigt und gab einen Punkteabzug.

\section{Schlussfolgerungen}

Die bisherigen Studien zeigen, dass von selbstständig ausgeführten Atemübungen in der Regel keine Verschlechterungen zu erwarten sind - dies gilt auch für die behandlungsinduzierten, durch den Therapeuten durchgeführten atemtherapeutischen Interventionen, bei denen bei qualifizierter Diagnosestellung und kompetenter Arbeitsweise in der Regel keine Nebenwirkungen zu erwarten sind. Bei der Atemtherapie handelt es sich also um eine sichere Methode.

Auch wenn einzelne der zur Atemtherapie vorliegenden Studien gewisse methodische Mängel aufweisen, so sind die Hinweise doch stichhaltig und überzeugend, dass atemtherapeutische Methoden sowohl somatisch als auch psychisch wirksam sein können und sicher und schonend in der Anwendung sind. 


\section{Danksagung}

Unser besonderer Dank gilt Herrn Prof. Reinhard Saller von der Universität Zürich und Herrn Prof. Wolfgang Tschacher von der Universität Bern für die wissenschaftliche Betreuung dieses Reviews.

\section{Disclosure Statement}

Wir erklären, dass wir im Zusammenhang mit der Durchführung dieses wissenschaftlichen Forschungsprojekts und dem oben beschriebenen Review keinerlei Interessenkonflikt haben. Dieses Forschungsprojekt erhielt keinerlei Zuschuss oder Unterstützung von irgendeinem Leistungsträger im öffentlichen, kommerziellen oder Non-Profit-Bereich.

\section{Literatur}

1 Bischof S: Atempädagogische/-therapeutische Tätigkei in der Bundesrepublik Deutschland: Ergebnisse einer empirischen Studie. Berlin, Arbeits- und Forschungsgemeinschaft für Atempflege AFA, 1991.

2 Büscher M: Beschreibung des atemtherapeutischen Prozesses anhand der Auswertung von Therapieverläufen: Eine qualitative Studie. Dissertation Fachbereich Psychologie, Technische Universität Berlin, 1993.

3 Büscher M, Weiss A: Kann Atemtherapie heilen? Eine Auswertungsstudie. Berlin, Arbeits- und Forschungsgemeinschaft für Atempflege AFA, 1994.

4 Dietrich S: Atemrhythmus und Psychotherapie: Ein Beitrag zur Geschichte der Psychosomatik und ihrer Therapien. Med. Dissertation, Universität Bonn, 1995.

5 Heyer-Grothe L: Über Hilfsmethoden der Psychotherapie, Gymnastik, Atmung, Massage; in Heyer GR (ed): Praktische Seelenheilkunde. München, Lehmanns, 1935; Nachdruck: München, Kindler, o.J.

6 Heyer-Grothe L (ed): Atemschulung als Element der Psychotherapie. Darmstadt, Wissenschaftliche Buchgesellschaft, 1970.

7 Mehling WE: Atemtherapie: Der gegenwärtige Stand der Atemtherapie in Deutschland; ihre Stellung zur Medizin und ihre Anwendung bei Rückenschmerzen. Dissertation, Freie Universität Berlin, 1999.

8 von Steinaecker K: Luftsprünge: Anfänge moderner Körpertherapien. München, Urban \& Fischer, 2000.

9 Stutz R, Anke K, de Roche F: Atemtherapie als salutogenes Heilverfahren: Ein Konzept zur Aktivierung der Selbstheilkräfte in Prävention und Therapie. Schweiz Z Ganzheitsmed 2006;18:159-162.

10 Mehling WE: Atemtherapie: Grundlagen, Wirkungsweisen, Interventionsstudien; in Müller-Braunschweig H (ed): Körperorientierte Psychotherapie: Methoden Anwendungen - Grundlagen. Berlin, Springer, 2010, pp 158-173.

11 Fischer K, Kemmann-Huber E: Der bewusste zugelassene Atem: Theorie und Praxis der Atemlehre. München, Urban \& Fischer, 1999.

12 Meier S: Atemtherapie nach Middendorf in der Schweiz: Eine wissenschaftliche Bestandesaufnahme aus Patientensicht. Dissertation, Universität Zürich, 2002.

13 Middendorf I: Der Erfahrbare Atem: Eine Atemlehre, ed 4. Paderborn, Junfermann, 1987.

14 Richter H: Atemwelten. Wiesbaden, Dr. Ludwig Reichert Verlag, 2005.

15 van Dixhoorn J: Body Awareness: the proper application of relaxation and breathing technique. Gedrag (Psychol Health) 1984;12-15:31-45.

16 Wolf K: Integrale Atemschulung. Bern, Humata, 1994.

17 Lehnert-Schroth C: Dreidimensionale Skoliosetherapie, ed 8. München, Berlin, Urban \& Fischer, 2014.

18 Courtney R, Cohen M: Investigating the claims of Konstantin Buteyko, M.D., Ph.D.: the relationship of breath holding time to end tidal $\mathrm{CO}_{2}$ and other proposed measures of dysfunctional breathing. J Alternat Complement Med 2007;14:115-123.
19 Malin L: Der Einfluss der Atemtherapie auf die menschliche Stimme: Vergleichsstudie anhand der technischen Stimmmessung bei Gesangsstudentinnen. Berlin, LIT Verlag, 2009.

20 Fürst K: Atem- und Körperpsychotherapie: Grundlagen und Therapie; in Bischof S (ed): Atem der ich bin. Atem- und Körperpsychotherapie bei frühen Störungen. Norderstedt, Books on Demand, 2012, pp 69-120.

21 Rieder A: Atempsychotherapie als körperpsychotherapeutisches Verfahren; in Bischof S (ed): Atem der ich bin. Atem- und Körperpsychotherapie bei frühen Störungen. Norderstedt, Books on Demand, 2012, pp 311-350

22 Moher D, Liberati A, Tetzlaff J, Altmann DG; The PRISMA Group: Preferred reporting items for systematic reviews and meta-analyses: the PRISMA statement. PLoS Med 2009;6:e1000097.

23 Both A: Brimful of STARLITE: towards standards for reporting literature searches. J Med Library Assoc 2006; 94:421-429.

24 Higgins J: Cochrane Handbook for Systematic Reviews of Interventions (Version 5.1.0). Cochrane Collaboration, http://handbook.cochrane.org/, 2011 (letzter Zugriff 20. Februar 2015)

25 Aust G, Fischer K: Veränderungen von Körpergleichgewichtsreaktionen durch Atemarbeit: Eine posturographische Studie unter Einbeziehung des visuellen Feedbacks. Laryngorhinootologie 1997;76:577-582.

26 Bowler S, Green A, Mitchell C: Buteyko breathing techniques in asthma: a blinded randomised controlled trial. Med J Aust 1998;169:575-578.

27 Bruton A, Lewith GT: The Buteyko breathing for asthma: a review. Complement Ther Med 2005;13:4146.

28 Cooper S, Oborne J, Newton S, Harrison V, Thompson Coon J, et al: Effects of two breathing exercises (Buteyko and Pranayama) in asthma: a randomised controlled trial. Thorax 2003;58:674-679.

29 Cowie RL, Conley DP, Underwood MF, Reader PG: A randomised controlled trial of the Buteyko technique as an adjunct to conventional management of asthma. Respir Med 2008;102:726-732.

30 Daubenmier J, Sze J, Kerr CE, Kemeny ME, Mehling WE: Follow your breath: respiratory interoceptive accuracy in experienced meditators. Psychophysiology 2013;50:777-789.

31 Eckert B: Atemtherapie bei Palliativpatienten. Dissertation, Ludwig-Maximilians-Universität München, 2014.

32 Goetz K, Loew T, Hornung R, Cojocaru L, Lahmann C, Tritt K: Primary prevention programme for burnoutendangered teachers: follow-up effectiveness of a combined group and individual intervention of AFA breathing therapy. Evid Based Complement Alternat Med 2013;2013:798260.
33 Holloway EA, West R: Integrated breathing and relaxing training (the Papworth method) for adults with asthma in primary care: a randomised controlled trial. Thorax 2007;62:1039-1042.

34 Huber B, Böttcher S, Camerer C, Grün R: Atemtherapie im Rahmen eines Gruppeninterventionsprogramms für Angehörige von Kindern im Wachkoma Ergebnisse einer qualitativen Studie. Neuro Rehabil 2010;16:1-7.

35 Loew T, Götz K, Hornung R, Tritt K: Die AFA-Atemtherapie als Burnout Prophylaxe bei Lehrerinnen und Lehrern. Forsch Komplementmed 2009;16:174-179.

36 Mehling WE, Hamel KA, Acree M, Byl N, Hecht FM: Randomized, controlled trial of breath therapy for patients with chronic low-back pain. Altern Ther Health Med 2005;11:44-52.

37 Opat AJ, Cohen MM, Bailey MJ, Abramson MJ: A clinical trial of the Buteyko breathing technique in asthma as taught by a video. J Asthma 2000;37:557-564.

38 Prem V, Sahoo RC, Adhikari P: Comparison of the effects of Buteyko and Pranayama breathing techniques on quality of life in patients with asthma - a randomized controlled trial. Clin Rehabil 2012;27:133-141.

39 Slader CA, Reddel HK, Spencer LM, Belousova EG, Armour CL, et al: Double blind randomised controlled trial of two different breathing techniques in the management of asthma. Thorax 2006;61:651-656.

40 von Steinaecker K, Welke J, Bühring M, Stange R: Pilotuntersuchung zu atemtherapeutischem Gruppenunterricht bei Patienten mit Asthma bronchiale. Forsch Komplementmed 2007;14:86-91.

41 van Dixhoorn J, Duivenvoorden HJ, Staal JA, Pool J, Verhage F: Cardiac events after myocardial infarction: possible effect of relaxation therapy. Eur Heart J 1987;8: 1210-1214.

42 van Dixhoorn J, Duivenvoorden HJ: Breathing awareness as a relaxation method in cardiac rehabilitation; in McGuigan WE, Macdonald Wallace J (eds): Stress and Tension Control 3. New York, Plenum Press, 1989, pp 19-36.

43 van Dixhoorn J, Duivenvoorden HJ, Pool J, Verhage F: Psychic effects of physical training and relaxation therapy after myocardial infarction. J Psychosom Res 1989; 34:327-337.

44 van Dixhoorn J, Duivenvoorden HJ, Staal HA, Pool J Physical training and relaxation therapy in cardiac rehabilitation assessed through a composite criterion for training outcome. Am Heart J 1989;118:545-552.

45 van Dixhoorn J: Cardiorespiratory effects of breathing and relaxation instruction in myocardial infarction patients. Biol Psychol 1998;49:123-135.

46 Davidson R, Kabat-Zinn J, Schumacher J, Rosenkranz $\mathrm{M}$, Muller D, et al: Alterations in brain and immune function produced by mindfulness meditation. Psychosom Med 2003;65:564-570. 